\title{
BMJ Open Diagnostic evaluation for autism spectrum disorder: a survey of health professionals in Australia
}

\author{
Lauren J Taylor, ${ }^{1,2}$ Valsamma Eapen, ${ }^{2,3}$ Murray T Maybery, ${ }^{1,2}$ Sue Midford, ${ }^{4}$ \\ Jessica Paynter, ${ }^{2,5,6}$ Lyndsay Quarmby, ${ }^{7}$ Timothy Smith, ${ }^{2,8}$ Katrina Williams, ${ }^{2,9,10,11}$ \\ Andrew J O Whitehouse ${ }^{2,12}$
}

To cite: Taylor LJ, Eapen V, Maybery MT, et al. Diagnostic evaluation for autism spectrum disorder: a survey of health professionals in Australia. BMJ Open 2016;6: e012517. doi:10.1136/ bmjopen-2016-012517

- Prepublication history and additional material is available. To view please visit the journal (http://dx.doi.org/ 10.1136/bmjopen-2016012517).

Received 10 May 2016 Revised 11 August 2016 Accepted 16 August 2016

CrossMark

For numbered affiliations see end of article.

Correspondence to

Dr Lauren Taylor;

lauren.taylor@kcl.ac.uk

\section{ABSTRACT}

Objectives: There is currently no agreed Australian standard for the diagnosis of autism spectrum disorder (ASD) even though there are specific diagnostic services available. We suspected inconsistency in the diagnostic practices of health professionals in Australia and aimed to assess these practices across the nation by surveying all relevant professional groups.

Design: In this study, we completed a survey of 173 health professionals whose clinical practice includes participating in the diagnostic process for ASD in Australia. Participants completed an online questionnaire which included questions about their diagnostic setting, diagnostic practice and diagnostic outcomes in 2014-2015.

Participants: Participants covered a range of disciplines including paediatrics, psychiatry, psychology, speech pathology and occupational therapy. All states and territories of Australia were represented.

Setting: Participants came from a range of service settings which included hospitals, non-governmental organisations, publicly funded diagnostic services and private practice.

Results: There was variability in diagnostic practices for ASD in Australia. While some clinicians work within a multidisciplinary assessment team, others practice independently and rarely collaborate with other clinicians to make a diagnostic decision. Only half of the respondents reported that they include a standardised objective assessment tool such as the Autism Diagnostic Observation Schedule in ASD assessments, and one-third indicated that they do not include measures of development, cognition and language in assessments where ASD is suspected.

Conclusions: Reported practice of some professionals in Australia may not be consistent with international best practice guidelines for ASD diagnosis. These findings highlight the need for a minimum national standard for ASD diagnosis throughout Australia that ensures best practice regardless of the type of setting in which the service is provided.

In the absence of biomarkers, diagnosis of autism spectrum disorder (ASD) is based on the behavioural presentation of an

\section{Strengths and limitations of this study}

- This is the first national study to investigate the diagnostic practices of health professionals from different disciplines across Australia.

- While the study included clinicians from a range of professional backgrounds, there were an unequal number of respondents from each professional group.

- Respondents were self-selected and may be committed to high standards of clinical practice.

individual. The 'gold standard' in ASD diagnosis is a best estimate clinical diagnosis which is determined in accordance with current diagnostic classification systems and following rigorous assessment practices. There is general consensus that a rigorous diagnostic assessment for ASD would consist of a physical examination, hearing test, child observation and parent interview which includes a full developmental history (NICE, 2011). 'Best-practice' ASD assessments are more comprehensive, and also comprise standardised developmental or cognitive testing, language assessment and information from more than one setting, ideally from a source other than the parent or carer who has been interviewed. ${ }^{1-5}$ Rigorous assessments enhance the accuracy of diagnoses and provide information about an individual's strengths and difficulties which is important for intervention planning.

Despite the internationally recognised best practice guidelines for ASD diagnosis, ${ }^{3}$ and position statements from professional bodies $^{6-8}$ Australia does not have a national standard for ASD diagnosis. In addition, health services are governed on a state or territory basis, rather than on a national level. While in some jurisdictions, eligibility for publicly funded intervention for children with ASD requires a diagnosis made by a multidisciplinary team comprising a medical 
professional, psychologist and speech pathologist, other states have less stringent criteria, requiring only a paediatrician or a psychiatrist. Furthermore, eligibility for funding from the Helping Children with Autism funding package is determined following a conclusive ASD diagnosis which can be provided by a single paediatrician or a psychiatrist. ${ }^{9}$

Identifying ASD accurately and early is essential given that state and federal funding for early intervention is based on a formal diagnosis made before a child turns 7 years. ${ }^{9}$ An inaccurate diagnosis may mean that children are not eligible for early intervention which contains elements that specifically focus on areas of difficulty in ASD. ${ }^{10}$ Alternatively, children who are misdiagnosed with ASD may access services that are not relevant or effective for their areas of difficulty thereby wasting resources. In addition, it is well established that early intervention improves outcomes for children with $\mathrm{ASD}^{11} 12$ and that children who start intervention at a young age make more improvements than children who start at an older age. ${ }^{13-15}$ While ASD can be reliably diagnosed at two years, ${ }^{16-18}$ the average age of diagnosis in Australia is over 4 years (49 months), with ASD most frequently diagnosed at 71 months ${ }^{19}$ Variability in the age of diagnosis has been observed between Australian states, with significantly earlier age of diagnosis in Western Australia and New South Wales relative to all other states and territories. The variation in age at diagnosis may be associated with inconsistent diagnostic practices across the nation.

While three previous studies have investigated diagnostic practices for ASD in Australia, this research has included only paediatricians, ${ }^{20-24}$ psychiatrists $^{20} 2324$ and psychologists. ${ }^{24}$ Skellern $e t a l^{22}$ examined the assessment practices of paediatricians $(\mathrm{N}=79)$ and child psychiatrists $(\mathrm{N}=26)$ in Queensland, finding considerable variability in the diagnostic practices of these clinicians. ${ }^{2}$ The results of a more recent survey has shown similar findings. In a study of 124 paediatricians across Australia, Randall $e t a t^{20}$ found that only a minority of participating clinicians usually included information from cognitive/developmental assessments, or involved other disciplines in the diagnostic process. These practices are inconsistent with current clinical guidelines for ASD diagnosis. ${ }^{4}$ The lack of consistent standards in Australia likely results in different diagnostic protocols and variability in the quality and accuracy of ASD diagnoses.

To date, no national research has been conducted to investigate diagnostic practices for ASD across all health professions and throughout Australia. We aimed to include a sample of clinicians that covered a range of disciplines, states, geographic locations and service settings. In this study, we compared diagnostic practices for ASD across the Australian states and between clinicians who are experienced in the assessment and diagnosis of ASD. Given that there is no national standard for ASD diagnosis, it was hypothesised that there would be variability in diagnostic practices across states and between clinicians.
METHOD

\section{Participants}

We aimed to include responses from a range of professional groups, working across different clinical and geographical locations, to reflect the diversity of diagnostic assessment practice in Australia. Recruitment took a multifaceted approach: (1) The Cooperative Research Centre for Living with Autism (Autism CRC) website and social media platforms were used to advertise the research. The Autism CRC is a national cooperative research effort focused on ASD across the lifespan which includes universities, government agencies, service providers, Autism awareness groups and professional bodies; (2) relevant member bodies and professional organisations, including Speech Pathology Australia, the National Rural Health Alliance, the Western Australian Autism Diagnostician's Forum, the Neurodevelopmental and Behavioural Paediatric Society of Australasia and the Autism South Australia diagnostician's membership list, distributed information about the survey via mailing lists, newsletters and social media platforms; (3) psychologists with contact details listed on the Australian Psychological Society ASD Provider list were individually emailed and invited to participate in the research; (4) government-funded diagnostic services in each state were identified and the survey was either emailed or mailed to each service, along with a personalised letter inviting the service to complete the survey and (5) the researchers used individual networks to distribute the survey to colleagues. All states and territories, ${ }^{\mathrm{i}}$ as well as all disciplines included in multidisciplinary assessments for ASD were represented (see table 1).

There was a similar number of responses from the public/non-government $(\mathrm{n}=66,38 \%)$ and private $(\mathrm{n}=76$, $43 \%)$ sectors, with $31(19 \%)$ respondents working in the public and private sectors. ${ }^{\text {ii }}$ Public services and nongovernment organisations (NGOs) were grouped when participants provided background information. Respondents who indicated that they worked in the public sector or NGO $(n=38)$ were asked to specify whether this was a hospital $(n=27)$, government $(n=21)$ or NGO $(n=17)$. There were only eight completed responses obtained for health professionals working in NGOs, so the public/NGO grouping was retained throughout the data analysis.

A similar proportion of the respondents worked as sole practitioners $(n=49 ; 37 \%)$ and within a multidisciplinary team $(n=52 ; 39 \%)$, with $31(23 \%)$ respondents following 'other' models of practice, working as a sole

${ }^{\mathrm{i} T h e r e ~ a r e ~ s i x ~ A u s t r a l i a n ~ s t a t e s, ~ N e w ~ S o u t h ~ W a l e s ~(N S W), ~ S o u t h ~}$ Australia (SA), Victoria (Vic), Queensland (Qld), Tasmania (Tas) and Western Australia (WA), and two territories, the Australian Capital Territory (ACT) and the Northern Territory (NT).

${ }^{i i}$ Participants who reported that they had part-time employment in the public and the private sectors were asked to indicate which setting was their primary setting, that is, where they spend more than $50 \%$ of their time. All responses were then based on the primary practice setting. 
Table 1 Number of respondents from each state or territory of Australia, categorised by profession

\begin{tabular}{|c|c|c|c|c|c|c|c|c|c|}
\hline & \multicolumn{8}{|c|}{ Australian state/territory } & \multirow[b]{2}{*}{ Total } \\
\hline & $\overline{\mathrm{ACT}}$ & NSW & NT & QId & SA & Tas & Vic & WA & \\
\hline General practice & - & - & - & - & - & - & 1 & - & 1 \\
\hline Paediatrics & - & 7 & 1 & 4 & 8 & 3 & 5 & 4 & 32 \\
\hline Psychiatry & - & 2 & 1 & - & - & - & - & 1 & 4 \\
\hline Psychology & 2 & 13 & - & 9 & 14 & 6 & 14 & 17 & 75 \\
\hline Speech pathology & - & 3 & 3 & 2 & 15 & 4 & 11 & 8 & 46 \\
\hline Occupational therapy & - & 1 & - & 2 & 2 & - & 4 & - & 9 \\
\hline Other* & - & 2 & - & 1 & 1 & 1 & 1 & - & 4 \\
\hline Total & 2 & 28 & 5 & 18 & 40 & 14 & 36 & 30 & 173 \\
\hline
\end{tabular}

${ }^{*}$ Comprising one manager (TAS), researcher (QLD), dual paediatrics/psychiatry (VIC), autism consultant/researcher (SA) and not specified (NSW).

ACT, Austrlian Captial Territory; NT, Northern Territory; NSW, New South Wales; QLD, Queensland; SA, South Australia; Tas, Tasmania; Vic, Victoria; WA, Western Australia.

practitioner and within a multidisciplinary team (MDT). While there was no significant difference in the proportion of practitioners working in MDTs or as sole practitioners across states, $\chi^{2} \quad(5, \quad \mathrm{~N}=101)=10.16, \quad \mathrm{p}=0.07$, $\Phi=0.32$, there was a higher proportion of MDT practitioners in the public $(69 \%)$, relative to the private $(35 \%)$ sector, $\chi^{2}(1, \mathrm{~N}=101)=12.21, \mathrm{p}<0.001, \quad \Phi=0.35$. Conversely, a higher proportion of sole practitioners came from the private $(69 \%)$ relative to the public $(31 \%)$ sector.

Participants had been involved in ASD diagnosis for a median of 9.5 years $(\mathrm{SD}=7.50$ years, range $1-30$ years). Seventy-six participants $(85 \%)$ had completed training in ASD assessment. Training consisted of either undergraduate or postgraduate education, supervision, case discussions or participation in courses for the administration of the Autism Diagnostic Observation Schedule, ${ }^{25}$ Autism Diagnostic Interview-Revised ${ }^{26}$ or Developmental, Dimensional and Diagnostic Interview. ${ }^{27}$

\section{Procedure}

Participants completed an anonymous 141-item questionnaire that was presented via Qualtrics (Qualtrics, Utah, USA), an online survey platform. The survey was initially piloted by eight clinicians and researchers with expertise in ASD and revised based on feedback from this group prior to administration. The survey contained questions that fell into three categories: (1) diagnostic service, (2) diagnostic practice and (3) diagnostic outcomes (see online supplementary appendix A for more information on the types of questions included in the survey).

Background information, such as discipline, years of professional experience and type of training the respondent had participated in was also gathered. Questions were presented in a categorical format (Yes/ No), or on a seven-point Likert scale which ranged from 1 (Never) to 7 (Always). Some questions, such as the number of assessments completed in the past 12 months, required a numerical response. Respondents also provided some percentage responses, for example, the percentage of assessments in which they collaborate with other professionals. A copy of the questionnaire is available from the Autism CRC on request.

\section{Statistical analysis}

The survey was designed so that participants were only required to respond questions that were directly relevant to their practice. This resulted in different numbers of responses for different questions. Data were analysed based on the number of responses recorded for each question. All data were screened for normality prior to analysis. Non-parametric tests (Kruskal-Wallis and Mann-Whitney U) were used where the data were not normally distributed. Otherwise, continuous data were analysed using analysis of variance (ANOVA), with post hoc (Scheffe' test) comparisons used to follow-up significant omnibus ANOVA results. Categorical data were analysed using the chi-square test.

\section{RESULTS}

\section{Response and completion rates}

Two-hundred and forty-five potential participants opened the survey, with 173 of these individuals proceeding beyond the first question. Complete responses were obtained for 99 of the respondents who started the questionnaire, resulting in a completion rate of $58 \%$.

\section{Diagnostic processes}

Wait-list periods: The wait-list period for an ASD assessment was significantly longer in public/NGO relative to private settings (see table 2 ). While most of the private diagnostic services $(n=52,88 \%)$ start ASD assessments within 3 months of referral, wait times in the public sector were variable, with only 23 of the 50 respondents (46\%) reporting wait-list times of 3 months or less (see figure 1). The wait for an MDT assessment (median=12 weeks, $\mathrm{SD}=25.75$, range $=1.5-108$ weeks) was also longer than for a sole practitioner (median $=4$ weeks, $S D=9.84$, range $=1-52$ ), Mann-Whitney $\mathrm{U}=511, \mathrm{p}=0.005$, perhaps due to the higher number of MDTs in the public sector relative to the private sector. 
Table 2 Median (SD) and range for the wait-list period, number of assessment sessions and length of assessment sessions for diagnosticians in the private and public sectors

\begin{tabular}{|c|c|c|c|c|}
\hline & Private & Public & $\mathbf{U}$ & p Value \\
\hline \multicolumn{5}{|c|}{ Wait-list period (in weeks) } \\
\hline Median (SD) & $4(15.5)$ & $16(20)$ & 581 & $<0.001$ \\
\hline Range & $1-52$ & $2-108$ & & \\
\hline \multicolumn{5}{|c|}{ Assessment sessions } \\
\hline Median (SD) & $2(1.3)$ & $2(1.3)$ & 1072 & 0.056 \\
\hline Range & $0-6.5$ & $1-6.5$ & & \\
\hline \multicolumn{5}{|c|}{ Assessment length (in minutes) } \\
\hline Median (SD) & $90(88.6)$ & $120(108.8)$ & 1012 & 0.03 \\
\hline Range & $30-420$ & $40-600$ & & \\
\hline
\end{tabular}

Assessment sessions and length: Respondents $(\mathrm{n}=106)$ completed a median of 2 (range $=1-6.5$ ) sessions for ASD assessments with a median assessment length of $90 \mathrm{~min}$ ( $\mathrm{SD}=98.15 \mathrm{~min}$, range $30-600 \mathrm{~min}$ ). There was no difference in the number of assessment sessions completed in the private relative to the public sector (see table 2). However, assessment sessions in the public sector were significantly longer than those in the private sector.

Multidisciplinary assessment: MDTs $(\mathrm{n}=52)$ most commonly consisted of one medical and two allied health professionals, usually a psychologist and a speech pathologist. All of the participating occupational therapists were part of an MDT. Most MDTs conducted assessments either in series, together, partially together or in collaboration, that is, each clinician completes an independent assessment, but all assessors meet to make a consensus diagnostic decision (see figure 2). In contrast, sole practitioners $(n=49)$ tended to complete assessments in isolation or in series, that is, assess an individual independently one after the other. Only small numbers of the sole practitioners reported collaborating with other clinicians, with 1 of the $15(7 \%)$ sole practitioners working in isolation and 4 of those working in series $(17 \%)$ collaborating with external agencies.

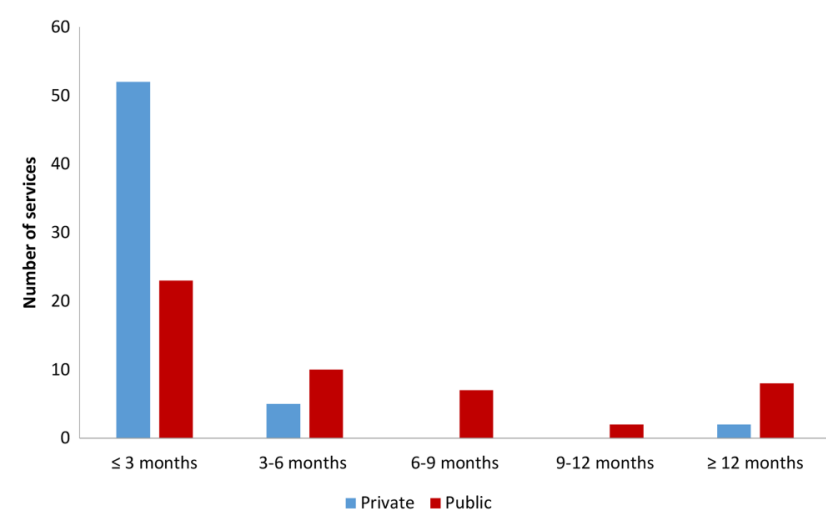

Figure 1 Total number of public and private services which have wait-list periods of $<3$ months to more than 12 months.

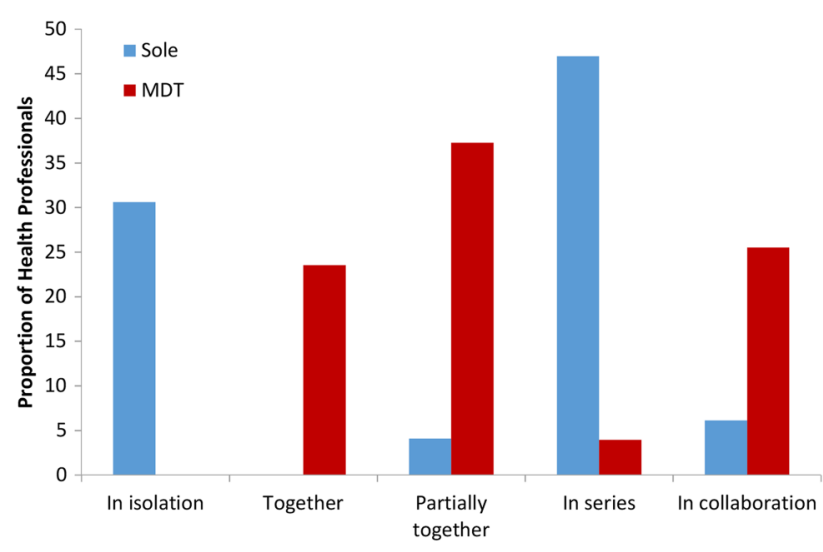

Figure 2 Proportion of sole practitioners and MDTs who conduct ASD assessments in isolation, together, partially together, in series or in collaboration with professionals from another discipline. ASD, autism spectrum disorder; MDT, multidisciplinary team.

Multisetting assessment: The majority of the 108 respondents (95\%) observed the individual in the clinic in all assessments (median frequency $=100 \%$ of assessments, $\mathrm{SD}=31 \%$, range $=0-100 \%)$. Assessments in the home or school/daycare settings were less frequent, with $47 \%$ of respondents including home observations (median frequency $=0 \%$ of assessments, $\mathrm{SD}=22 \%$, range $=0-100 \%$ ) and $77 \%$ of respondents including observations in the school or daycare (median frequency $=20 \%$ of assessments, $\mathrm{SD}=31 \%$, range $=0-100 \%$ ). Only two clinicians $(2 \%)$, both from the public sector, include clinic and school/daycare observations in more than $75 \%$ of the assessments, with 12 clinicians (11\%), 4 private practitioners and 8 from the public sector, completing observations in the clinic and home settings. Three respondents (3\%), 2 from the private sector and one from the public sector, observed a child in the home and school/daycare settings in more than $75 \%$ of the assessments.

\section{Diagnostic practices}

Hearing test: In the Australian clinical pathway, it would be expected that the paediatrician conducts a hearing assessment before referring a child to an allied health team for an ASD assessment. ${ }^{7}$ Of the paediatricians $(n=20)$ surveyed, only $2(10 \%)$ reported that a hearing test is included in all ASD assessments. Another 2 paediatricians $(10 \%)$ indicated that they rarely include a hearing test in ASD assessments, 5 reported occasionally or usually (ie, in $30-50 \%$ of assessments) and 11 paediatricians $(55 \%)$ reported to include a hearing test frequently or usually (ie, $70-90 \%$ of assessments) where ASD is suspected.

Medical investigations: All of the paediatricians who include medical investigations in ASD assessments reported completing a genetic screen, Fragile $\mathrm{X}$ test and neurological and physical examinations. Of the 21 paediatricians, 15 respondents $(71 \%)$, reported that they 
Table 3 Proportion of respondents ( $\mathrm{N}=105)$ from each discipline who administer developmental, cognitive, language, adaptive, ASD or psychometric assessments

\begin{tabular}{llllllc}
\hline & Developmental (\%) & Cognitive (\%) & Language (\%) & Adaptive (\%) & ASD (\%) & Other (\%) \\
\hline Paediatrics $(\mathrm{N}=21)$ & 23.81 & 28.57 & 28.57 & 19.05 & 57.14 & 0 \\
Psychiatry $(\mathrm{N}=3)$ & 0 & 0 & 0 & 33.30 & 66.67 & 0 \\
Psychology $(\mathrm{N}=51)$ & 35.29 & 70.59 & 29.41 & 72.55 & 90.20 & 39 \\
Speech pathology $(\mathrm{N}=25)$ & 20.00 & 20.00 & 92.00 & 24.00 & 64.00 & 12 \\
Occupational Therapy $(\mathrm{N}=3)$ & 33.33 & 33.33 & 33.33 & 66.67 & 66.67 & 0 \\
Other ( $\mathrm{N}=2)$ & 50 & 0 & 0 & 50 & 100 & 0 \\
\hline
\end{tabular}

Respondents were given a list of assessments that fitted within each category, but were also able to specify other measures that they administer. Example assessments within each category are described at the foot of the table.

Developmental assessments: Griffiths, Bayley, Mullen.

Cognitive assessments: WPPSI, WISC, WAIS, Leiter, UNIT, WNV.

Language assessments: CELF, CASL, CCC-2, CC-A, PLS.

Adaptive assessments: VABS, ABAS, ABS, Scales of Independent Behaviour.

ASD assessments: ADOS, ADI, 3Di, DISCO, CARS, M-CHAT, SCQ, ASSQ, ASRS, CAST, SRS.

Other assessments: Connor's, CBCL, SDQ, BRIEF, Depression, Anxiety.

ASD, autism spectrum disorder.

include medical investigations frequently or usually (more than $70 \%$ of their assessments), with only 4 pediatricians $(19 \%)$ including medical investigations in all assessments where ASD is suspected.

Assessment measures: A total of 107 participants responded to questions regarding the administration of assessment tools. A developmental history was reported to have been undertaken by $89 \%$ of these respondents. Of these, $66(62 \%)$ reported always administering standardised assessments in diagnostic evaluations for ASD, and $21(20 \%)$ reported doing so frequently or usually (in $70-90 \%$ of ASD assessments). There was no difference in the proportion of respondents who frequently administer assessments in private $(\mathrm{n}=51,88 \%)$ relative to public $(\mathrm{n}=36,77 \%)$ service settings, $\chi^{2}(1, \mathrm{~N}=87)=2.35$, $\mathrm{p}=0.13, \Phi=0.15$, nor were there differences in the proportion of respondents who administer assessments by state, $\chi^{2}(5, \mathrm{~N}=105)=2.95, \mathrm{p}=0.71, \Phi=.17$.

Participants who reported administering assessments $(n=105)$ were asked to indicate which types of assessments are included in diagnostic evaluations for ASD. Since some measures are restricted to particular disciplines, it was unsurprising that there was variability in the proportions of clinicians administering cognitive, language and adaptive assessments and measures of ASD symptomatology (see table 3 ). In addition, only 50 respondents (47\%) administer the ADOS and 41 (39\%) the ADI-R. Thirty-two respondents (30\%) use the ADOS and the ADI-R together in diagnostic evaluations for ASD.

Of the 105 respondents who indicated that they administer assessments as part of diagnostic evaluations for ASD, only $8(8 \%)$ reported that they complete an assessment battery comprising measures of developmental, cognitive, language, adaptive skills and ASD symptomatology. The numbers increased only marginally when we examined developmental and cognitive assessments separately, with 11 respondents (10\%) completing a developmental assessment in addition to measures of language, adaptive skills and ASD symptoms, and 14 (13\%) completing a cognitive assessment in addition to these other measures. Taking the profession-specific assessments separately showed that 27 psychologists (53\%) administered cognitive and adaptive assessments in addition to measures of ASD symptomatology, and 14 speech pathologists $(56 \%)$ administered language assessments in addition to measures of ASD symptomatology.

To account for clinicians who may review the results of assessments that are administered by other disciplines, we also asked participants to indicate whether they review assessment results. Sixty-seven (68\%) respondents reported that they review assessment results. There were no differences in the proportion of respondents from each profession, $\chi^{2}(4, \mathrm{~N}=98)=6.29, \mathrm{p}=0.18, \Phi=0.18$, or state; $\chi^{2}(5, \mathrm{~N}=98)=2.31, \mathrm{p}=0.80, \Phi=0.15$ who review the results of assessments. Finally, we investigated whether respondents who do not regularly administer assessments (ie, in $<30 \%$ of assessments), review assessment results instead. Six of the 17 respondents (35\%) who do not regularly administer assessments always review assessment results. A further 3 respondents (18\%) who do not regularly administer standardised assessments often review these results of assessments that have been administered in other settings.

Two respondents, both sole practitioners, reported that they do not administer standardised assessments in diagnostic evaluations for ASD. These respondents reported that they do not administer standardised measures because they are not part of everyday practice, or because they have already been administered at another service. In addition, one respondent reported that the standardised measures are not required because diagnostic decision-making is outlined in the Diagnostic and Statistical Manual Fifth Edition.

Assessment of co-occurring neurodevelopmental or mental health conditions: Only 23 of the 105 respondents (22\%) who administer standardised assessments include additional measures of behaviour or 
psychopathology. The majority of these clinicians were psychologists (see table 3 ).

Facing uncertainty in diagnosis: Forty-seven of the 97 (48\%) participants who responded to this question reported that they make provisional ASD diagnoses when faced with diagnostic uncertainty. There was no difference in the proportion of provisional diagnoses between the private and public settings, $\chi^{2}(1, \mathrm{~N}=97)=2.53, \mathrm{p}=0.11$, $\Phi=0.16$, or across the states, $\chi^{2}(7, \mathrm{~N}=97)=12.8, \mathrm{p}=0.08$, $\Phi=0.36$. Of the respondents who make provisional diagnoses, $34(72 \%)$ reported that this label is rarely used, with the remaining $5(11 \%)$ and $2(4 \%)$ reporting that provisional diagnoses are given occasionally and sometimes respectively. Provisional diagnoses were reportedly given when individuals demonstrated subthreshold symptoms, were very young or would benefit from intervention. Thirty-nine respondents (83\%) reported that they reassess individuals with provisional diagnoses within a mean of 13 months ( $\mathrm{SD}=7.96$ months).

From 94 respondents, $16(17 \%)$ reported that they have diagnosed ASD when the person did not meet full criteria for the condition. Fifteen of these respondents $(88 \%)$ reported that this has rarely occurred $(<10 \%$ of their assessments), with the remaining respondents indicating that it has been occasional (about 30\% of assessments). While there was no significant difference in the proportion of overdiagnosis coming from each state, $\chi^{2}$ (7, $\mathrm{N}=94)=2.41, \mathrm{p}=0.79, \Phi=0.16$, a significantly higher proportion of respondents who made a diagnosis when the individual did not meet criteria came from the private $(81 \%)$ relative to the public $(19 \%)$ sector, $\chi^{2}(1$, $\mathrm{N}=94)=4.50, \mathrm{p}=0.035, \Phi=0.22$. When asked about the reasons for making the ASD diagnosis, $14(88 \%)$ clinicians reported that they thought that the individual did have ASD, but that the assessment did not reveal the individual's actual diagnostic status. Other commonly reported reasons for the diagnosis were to ensure that the child could access early intervention $(n=6)$, school support $(n=5)$ or disability services $(n=3)$.

\section{DISCUSSION}

This study investigated the diagnostic practices for ASD across Australia. The results show inconsistencies in ASD assessment practices across the states, and between the private and public service settings. In addition, some clinicians in Australia may not be practising in a manner that is consistent with international best practice guidelines $^{4}$ or statements from Australian professional bodies ${ }^{7}$ for ASD assessment and diagnosis.

The current findings are consistent with the results reported by Randall et $a l,{ }^{20}$ who found that only a minority of Australian paediatricians follow current recommendations for diagnosing ASD. Comprehensive diagnostic assessments that comprise all of the recommended elements are lengthy and expensive, so it is possible that the time required to administer standardised assessments of ASD symptomatology in addition to conducting observations of the individual across settings is prohibitive for practitioners.

A small proportion of respondents (17\%) reported that they have diagnosed ASD when a person does not meet the criteria for the disorder. This was more likely to happen in the private sector than in the public sector. The reasons for making this diagnosis included the assessment did not adequately show the actual diagnostic status or to ensure that the person could access early intervention, support at school or access other disability services. These findings are consistent with previous research that has investigated the diagnostic practices of clinicians in Queensland, the USA and the UK. ${ }^{21} 2328$ The results of these previous studies have indicated that up to $55 \%$ of participating health professionals had increased the level of diagnosis or severity, most commonly because of a lack of diagnostic certainty, but also to ensure that the individual could access funding, educational support or other services. The intentional ascription of an inaccurate diagnosis to facilitate access to services may contribute to the increased diagnostic rates over time. Indeed, Nassar $e t a l^{29}$ found that the increased incidence of ASDs in WA was related to changes in diagnostic practices and service provision in this state. These findings highlight the complexity of ASD diagnosis and the challenges associated with funding being designated according to diagnosis rather than need.

While the sample size in this study was small, the response rate is consistent with previous research indicating that the average response rate for surveys is $55.6 .{ }^{30}$ The number of completed responses in our survey is also consistent with similar surveys conducted in the UK and Australia. ${ }^{24} 28$ In particular, Ward $e t a l^{24}$ included 104 psychologists, psychiatrists and paediatricians. While the number of responses in this study was similar to Ward et al, our survey includes a wider range of disciplines and covers a greater number of Australian states and territories. Furthermore, our results are consistent with several smaller surveys of Australian paediatricians and psychiatrists, which reported variations in assessment and diagnostic practices between clinicians and across professional groups. ${ }^{22} 23$

Although our sample had representatives from all states and relevant professions, the numbers were not consistent across categories. In addition, respondents in this study were self-selected, perhaps because of a strong interest in good diagnostic standards, so the results may not represent the broader population of diagnosticians in Australia. Notwithstanding this, the findings suggest the need for the development of national standards in ASD diagnostic practices and the subsequent evaluation of these standards.

\section{CONCLUSION}

This research is the first study to investigate diagnostic practices for a range of professionals across Australia. We found that a number of health professionals are conducting 
assessments that are inconsistent with best practice guidelines. This may contribute to poor quality assessments and impact estimates of ASD prevalence in Australia. Assessments that do not comply with best practice guidelines may also lead to delayed diagnosis and access to intervention services. Conversely, assessments that more closely resemble current clinical guidelines for ASD diagnosis may be costly and associated with longer wait-list periods. Therefore, there is a need to balance the quality and cost of ASD assessments to ensure that ASD diagnoses are conducted in a thorough but efficient manner. In addition, findings regarding the possible overdiagnosis of ASD have implications for service delivery, particularly when funding and service provision is determined based on diagnosis. These findings have implications for future policy, particularly with regard to establishing a national standard in ASD diagnosis, to ensure an equitable process for families and individuals with ASD.

\section{Author affiliations}

${ }^{1}$ School of Psychology, The University of Western Australia, Crawley, Western Australia, Australia

${ }^{2}$ Cooperative Research Centre for Living with Autism (Autism CRC), Brisbane, Queensland, Australia

${ }^{3}$ University of New South Wales, New South Wales, Australia

${ }^{4}$ Western Australian Autism Diagnostician's Forum, Western Australia, Australia

${ }^{5}$ Research Department, AEIOU Foundation, Queensland, Australia

${ }^{6}$ School of Applied Psychology, Griffith University, Gold Coast, Queensland, Australia

${ }^{7}$ Centre for Rural Health, University of Tasmania, Australia

${ }^{8}$ Disability Services Commission, Western Australia, Australia

${ }^{9}$ Department of Paediatrics, University of Melbourne, Melbourne, Victoria,

Australia

${ }^{10}$ Developmental Medicine, Royal Children's Hospital, Melbourne, Victoria,

Australia

${ }^{11}$ Murdoch Children's Research Institute, Melbourne, Victoria, Australia

${ }^{12}$ Telethon Kids' Institute, The University of Western Australia, Australia

Acknowledgements The authors also acknowledge the health professionals who dedicated their time to participate in this research.

Contributors LJT designed and administered the survey for health professionals, was involved in data analysis and interpretation and wrote and reviewed the manuscript. VE, MTM, SM, JP, LQ, TS, KW and AJOW were involved in the study design, including survey design and these authors also contributed to the preparation of the manuscript. All authors contributed to participant recruitment.

Funding The authors acknowledge the financial support of the Cooperative Research Centre for Living with Autism (Autism CRC), established and supported under the Australian Government's Cooperative Research Centres Programme and the Department of Social Services. Financial support was also obtained from the Commonwealth Department of Social Services.

Competing interests None declared.

Ethics approval This study was approved by the Ethics Committee of the University of Western Australia (Ref RA/4/1/6997).

Provenance and peer review Not commissioned; externally peer reviewed.

Data sharing statement No additional data are available.

Open Access This is an Open Access article distributed in accordance with the Creative Commons Attribution Non Commercial (CC BY-NC 4.0) license, which permits others to distribute, remix, adapt, build upon this work non- commercially, and license their derivative works on different terms, provided the original work is properly cited and the use is non-commercial. See: http:// creativecommons.org/licenses/by-nc/4.0/

\section{REFERENCES}

1. Anagnostou $E$, Zwaigenbaum L, Szatmari $P$, et al. Autism spectrum disorder: advances in evidence-based practice. Can Med $J$ 2014;186:509-19.

2. Baird G, Cass $H$, Slonims V. Diagnosis of autism. BMJ 2003;327:488-93.

3. Filipek PA, Accardo PJ, Baranek GT, et al. The screening and diagnosis of autism spectrum disorders. J Autism Dev Disord 2009;29:439-84.

4. NICE. Autism in under 19s: recognition, referral and diagnosis. 2011 https://www.nice.org.uk/guidance/cg128 (accessed 28 Nov 2015).

5. Ozonoff S, Goodlin-Jones BL, Solomon M. Evidence-based assessment of autism spectrum disorders in children and adolescents. J Clin Child Adolesc 2005;34:523-40.

6. Australian Advisory Board on Autism Spectrum Disorders. Position paper on the diagnosis and assessment of Autism Spectrum Disorders in Australia. http://www.autismadvisoryboard.org.au/ uploads/Position\%20Paper\%20on\%20Diagnosis\%20July\%202011\% 20Final.pdf (accessed 28 Jan 2016).

7. Silove N, Blackmore R, Warren A, et al. A consensus approach for the paediatrician's role in the diagnosis and assessment of autism spectrum disorders in Australia. https://www.racp.edu.au/docs/ default-source/advocacy-library/pa-pol-a-consensus-approach-forthe-paediatricians-role-in-the-diagnosis-and-assessment-of-autism. pdf (accessed 28 Jan 2016).

8. Glasson EJ, MacDermott S, Dixon G, et al. Management of assessments and diagnoses for children with autism spectrum disorders: the Western Australian model. Med J Aust 2008;188: 288-91.

9. Australian Government. Disability and Carers: Helping Children with Autism (HCWA) Eligibility. https://www.dss.gov.au/ourresponsibilities/disability-and-carers/program-services/for-peoplewith-disability/is-my-child-eligible (accessed 12 Nov 2015).

10. Prior M, Roberts JMA, Rodger S, et al. A review of the research to identify the most effective models of practice in early intervention for children with autism spectrum disorders. Australian Government Department of Families, Housing, Community Services and Indigenous Affairs, Australia, 2011. https://www.dss.gov.au/ourresponsibilities/disability-and-carers/program-services/for-peoplewith-disability/early-intervention-for-children-with-autism-spectrumdisorders-guidelines-for-good-practice-2012 (accessed 28 Jan 2016).

11. Magiati I, Tay XW, Howlin P. Early comprehensive behaviourally based interventions for children with autism spectrum disorders: a summary of findings from recent reviews and meta-analyses. Neuropsychiatry 2012;2:543-70.

12. Reichow B. Overview of meta-analyses on early intensive behavioral intervention for young children with autism spectrum disorders. $J$ Autism Dev Disord 2012;42:512-20.

13. Fenske EC, Zalenski S, Krantz PJ, et al. Age at intervention and treatment outcome for autistic children in a comprehensive intervention program. Anal Intervent Dev Disabil 1985;5:49-58.

14. Granpeesheh D, Dixon DR, Tarbox J, et al. The effects of age and treatment intensity on behavioural intervention outcomes for children with autism spectrum disorders. Res Autism Spectr Disord 2009;3:1014-22.

15. Rogers SJ, Estes A, Lord C, et al. Effects of a brief Early Start Denver Model (ESDM)-based parent intervention on toddlers at risk for autism spectrum disorders: a randomized controlled trial. J Am Acad Child Adolesc Psychiatry 2012;51:1052-65.

16. Cox A, Klein K, Charman T, et al. Autism spectrum disorders at 20 and 42 months of age: stability of clinical and ADI-R diagnosis. J Child Psychol Psyc 1999;40:719-32.

17. Moore V, Goodson S. How well does early diagnosis of autism stand the test of time? Autism 2003;7:47-63.

18. Eaves $\mathrm{LC}, \mathrm{Ho} \mathrm{HH}$. The very early identification of autism: outcome to age 41/2 -5. J Autism Dev Disord 2004;34: 367-78.

19. Bent CA, Dissanayake C, Barbaro J. Mapping the diagnosis of autism spectrum disorders in children aged under 7 years in Australia, 2010-2012. Med J Aust 2015;202:317-20.

20. Randall M, Albien-Urious N, Brignell A, et al. Diagnosing autism: an Australian paediatric research network survey. J Paediatr Child Health 2016;52:11-17. 
21. Rushton JL, Felt BT, Roberts MW. Coding of pediatric behavioral and mental disorders. Pediatrics 2002; 110:1-6.

22. Skellern $\mathrm{C}, \mathrm{McD}$ owell M, Schluter P. Diagnosis of autistic spectrum disorders in Queensland: variations in practice. J Paediatr Child H 2005;41:413-18.

23. Skellern C, Schluter P, McDowell M. From complexity to category: responding to diagnostic uncertainties of autistic spectrum disorders. $J$ Paediatr Child Health 2005;41:407-12.

24. Ward SL, Sullivan KA, Gilmore L. Practitioner perceptions of the assessment and diagnosis of Autism in Australia. Aust Psychol 2016;51:272-9.

25. Lord C, Rutter M, DiLavore $\mathrm{P}$, et al. Autism diagnostic observation schedule, second edition (ADOS-2). Torrance, CA: Western Psychological Services, 2012.
26. Rutter M, LeCouter A, Lord C. Autism diagnostic interview, revised (ADI-R). Torrance, CA: Western Psychological Services, 2003.

27. Skuse D, Warrington R, Bishop D, et al. The developmental, dimensional and diagnostic interview (3di): a novel computerised assessment for autism spectrum disorders. J Am Acad Child Adolesc Psychiatry 2004;43: 548-58.

28. Rogers CL, Goddard L, Hill EL, et al. Experiences of diagnosing autism spectrum disorder: a survey of professionals in the United Kingdom. Autism. Published Online First: 16 Dec 2015. doi:10.1177/ 1362361315611109

29. Nassar N, Dixon G, Bourke J, et al. Autism spectrum disorders in young children: effect of changes in diagnostic practices. Int J Epidemiol 2009;38:1245-54.

30. Baruch $Y$. Response rate in academic studies-a comparative analysis. Hum Relat 1999;52:421-38. 